while I can see very little advantage to the student in having the book held together by a plastic ringbinder, the advantage to the publisher is obviously enormous. After one year of use the book falls to pieces and the owner cannot recoup some of his outlay by selling it to those who follow. The quest to reduce the second-hand value is also helped by including "data sheets" and graph paper (with marked axes!) in the text. This surely spoonfeeds students too much, for one of the objectives of laboratory exercises is to introduce them to the craft of tabulating results and planning graphs.

The other two books reviewed here both deal with our Solar System. Lewis and Prinn have provided, in Planets and Their Atmospheres, the ideal starter pack. It is not for the faint hearted, however, being aimed accurately at the final-year student, postgraduate and research worker. To this end the authors have assembled an impressive 70 pages of references and a detailed, itemized index which has at most five page numbers for each topic. The book is also timely. Spacecraft have provided an enormous bank of data about the present atmospheres of the terrestrial planets Venus, Earth and Mars. Observations of Jupiter, Saturn, Uranus, Neptune, Titan and Io have also helped us formulate models for the structure, composition and chemistry of their gaseous mantles. Detailed comparative studies have allowed us to move away from the present state to a discussion of origins and evolution.

Why do I call it a "starter pack"'? Well, when considering a new topic, all researchers and students need to read a few sections of a good book to familiarize themselves with the relevant background before turning to shelves of journals in the library. There are too few good books of this type around. We must be grateful to Lewis and Prinn for providing another.

Jones's The Solar System is a nonmathematical introduction to our planets. I found it rather difficult to visualize the audience it is intended for. We are given a contemporary picture of the planets, with many references to the new data obtained from spacecraft, but the view has been somewhat drained of its excitement. Everything is mentioned, but only briefly and sketchily, and the author has thus given himself little room to point out the problems and mysteries. He has also taken the rather old-fashioned approach of compartmentalizing each planet into a separate chapter, which militates against fruitful comparisons. And now that we are so used to pages of colour images of planets, the reversion to black-and-white produces a sense of disappointment.

There are many general books presently available which deal with the Solar System. Unfortunately, most of them are better than this one.

David $W$. Hughes is Senior Lecturer in Astronomy and Physics at the University of Sheffield.

\title{
From eye to CCD
}

\section{Michael Rowan-Robinson}

\section{Astrophysical Techniques.}

By C.R. Kitchin.

Adam Hilger: 1984. Pp.438. Hbk £40,

$\$ 65 ; p b k £ 15, \$ 25$.

IN THE 50 years since Jansky first detected the Milky Way at radio wavelengths there has been a phenomenal explosion in techniques of astronomical observation. The new wavelengths - radio, X-ray, ultraviolet, infrared, microwave, gamma-ray and the other types of astronomical information reaching the Earth, such as neutrinos, cosmic rays and gravitational radiation, have each required new detection techniques, new types of telescope and new observing platforms, ranging from caverns deep under the Earth to satellite-borne telescopes. Optical astronomy too is being revitalized by new techniques, for example the CCD (charge-coupling device) array and the fibre-optics spectroscope.

Many university and polytechnic departments of physics or astronomy give courses on astronomical observing techniques and instrumentation. Not all of these bear much relation to what is actually happening in astronomy today, and there is a great need for authoritative and accessible books

\section{Inside the planets}

\section{D.C. Tozer}

Physics of Planetary Interiors.

By G.H.A. Cole.

Adam Hilger: 1984. Pp.208. Hbk £22, $\$ 39 ; p b k £ 9.95, \$ 16$.

THERE will always be a need for books that try to put the enormous complexity of the known planetary structure and behaviour into a readily comprehended form - books that show a student how much can be explained by a relatively straightforward application of generally accepted physical principles. Professor Cole's book is directed at undergraduates and his choice of subject and approach reflect his background in applied mathematics. For example, much - in my view, too much - attention is given in this introductory text to the gravitational fields and shapes of rotating massive bodies in hydrostatic equilibrium (though who would now deny that a world of meaning and whole fields of research have been inspired by the very small but definite departures of the actual geopotential surfaces from such predicted shapes?). A student needs much more guidance than he is given here for pursuing calculations that tacitly imply a very static view of planetary evolution.

Characteristically, an attempt is made to define the realm of planetary science as that on the subject. Dr Kitchin has set out to provide an account suitable for the sciencebased undergraduate, though he hopes that the amateur astronomer will find it useful and also points out that increasing specialization in the subject means that most professional astronomers would also benefit from such a book.

Dr Kitchin concentrates on techniques of detection and imaging, but extends this to include the basic optics used in modern telescopes. There is little discussion of the atmospheric and other background limitations which pose such a challenge to observational astronomy and which drive so many of the technical developments. The book is crammed with useful detail, though. It is strongest on the techniques of optical observation: the coverage of the new astronomies such as radio and $\mathrm{X}$-ray is fairly skeletal. Microwave and submillimetre developments are not mentioned. The physical basis of the techniques is briefly discussed, but a fair knowledge of atomic physics and optics is implicitly assumed.

This will be a useful addition to the reading list for a more advanced course on astronomical techniques. But the amateur astronomer may find it a bit heavy going.

Michael Rowan-Robinson is Reader in Astronomy at Queen Mary College, University of London. of objects in which self-gravitation is strong enough to induce an axial symmetry of their external shape, but not so strong as to make the atoms "incapable of maintaining the equilibrium which defines a planetary body". Such efforts at classification appeal to the tidy mind, but are likely to prove very confusing to the able and inquisitive student. "Minor planet" is a well-established usage for objects whose light curve suggests a highly irregular shape, and if the quotation I have taken is intended to mean no pressure ionization of atoms, what is one to call an object such as Jupiter, in which a substantial mass fraction is believed to be a pressure-induced metallic phase? Further clarification is not helped by the erroneous comment that free electrons are non-relativistic in planets but fully relativistic in white dwarf stars.

Unfortunately, this kind of ambiguous or poorly reasoned remark is all too common - I noticed serious conceptual errors and typographical mistakes that make the sections on planetary heat transfer and magnetism positively misleading. If there is no treatment of seismology, a chapter devoted to a hydrostatic theory of the structure of icy major planet satellites is an original feature. Nevertheless, I would not recommend this book to students until several corrections have been made.

D.C. Tozer is a Reader in Theoretical Geophysics at the University of Newcastle upon Tyne. 